\title{
Fine Binocular Control in Dyslexic Children
}

\author{
J. F. STEIN, * P. M. RIDDELL* and M. S. FOWLER $\dagger$ \\ Oxford and Reading
}

\begin{abstract}
Summary
Dyslexic children often complain that letters seem to move around. The hypothesis advanced here is that this is a symptom of immature vergence control which leads to an unstable sense of visual direction. Evidence is presented that

(1) sixty-seven per cent of dyslexic children exhibit poor dynamic control of vergence movements in response to a small fusion stimulus,

(2) most good readers have good vergence control,

(3) children with poor vergence control have reduced stereoacuity,

(4) six months monocular occlusion for reading and close work assisted 51 per cent of dyslexics with unstable vergence control to improve; thereafter their reading improved rapidly also.

It is concluded that defective vergence control is an important, though not the only, cause of dyslexics' problems.
\end{abstract}

The number of children who experience unexpected difficulties with learning to read is remarkably high. Estimates of the incidence of specific reading disability (reading age significantly behind that expected from performance IQ measures-developmental dyslexia) range from 4-20 per cent of 8-11 year olds. ${ }^{1.2}$ Four times as many boys as girls are affected, and in a high proportion of cases there is a family history of learning disabilities. In the small number of known dyslexics whose brains have been examined post mortem cortical abnormalities have been found, particularly in the language areas of the left hemisphere. ${ }^{3}$ These features imply that developmental dyslexia has an organic neurological basis. However, this conclusion is unpopular with many of those who deal with children with reading difficulties. It is taken to imply that the conditions must be irreversible if it is the result of a neurological disorder. Thus it would consign these children to permanent retardation. Unfortunately, it is clear from many studies that dyslexic problems do indeed persist into adulthood. Intelligent sufferers develop many ingenious ways of overcoming their deficits; but failure to read until after the age of 12 years, and even then slowly, probably leaves a permanent gap in verbal experience, and memory. However, these unpalatable facts should not be used to attempt to refute the evidence that developmental dyslexia is the result of organic brain dysfunction, but to encourage attempts to understand more about it in the hope of alleviation. One promising line of approach is to study the 'visual' symptoms that many dyslexics suffer.

Dyslexic children are often referred to eye departments because they complain that they cannot see properly. Very seldom is it found that they have any abnormality of the eye or retina. Their visual acuity is usually normal. In any case many children with very poor visual acuity learn to read perfectly well. On further questioning, however, one very often

From: * University Laboratory of Physiology, Oxford.

† Ophthalmology Department, Royal Berks Hospital, Reading.

Correspondence to: Dr. J. F. Stein, University Laboratory of Physiology, Oxford. 
finds that what these children are really experiencing is that their visual world becomes unstable when they try to fixate small letters. These seem to blur, swim around and jump over each other so that the child cannot determine their proper order.

Stable visual localisation requires that the retinal image of what is being foveated is accurately associated with ocular motor signals which indicate the direction in which the eyes are pointing at the time. This process provides the visual location of the object with respect to the observer, by calibrating its retinal locus in terms of the direction in which the fovea is pointing. However, to determine the direction in which the eyes are pointing is particularly difficult when they are converged for inspecting a small target, such as a letter, at a close distance. Although the required coarse angles of vergence and version are set by binocular disparity and retinotopic cues respectively, fine control of vergence demands that each eye be able to make adjustments independently of the other in order to keep the target on the fovea. Small ( $<1$ degree) vergence errors are the rule whenever normal subjects make conjugate or vergence eye movements. In normal subjects these are speedily corrected. Binocular retinal signals are disentangled so that the foveal signals provided by each eye can be fed back to control that eye's movements and point it accurately at the target. But this may not be true of dyslexics.

We therefore postulated that dyslexic children might have a deficiency of fine binocular control $^{4}$ which might explain their symptoms of perceptual instability when attempting to read. Poor vergence control would lead to inability to localise or spatially sequence small objects, including letters, properly. Hence it would explain why letters and words appear to blur, move around and change their order and why these children have such difficulty with learning to read.

Our hypothesis was originally developed from studying over a thousand normal and dyslexic children ${ }^{5.6 .7}$ using the Dunlop synoptophore test. ${ }^{8}$ In this test the child is required to make accurate divergent eye movements in response to a small (macular sized) fusion target while the synoptophore tubes are slowly diverged. If he has inaccurate vergence control such a child is unable to make reliable judgements of the visual direction of monocular controls; and it is the consistency of these subjective responses of the child which is recorded over 10 trials. Using the Dunlop test we have shown that between 60 and 70 per cent of dyslexic children give unreliable and inconsistent responses in this test; i.e. they demonstrate unstable vergence control. ${ }^{9}$ In contrast few good readers exhibit this abnormality. These results have recently been confirmed by Bigelow and McKenzie. ${ }^{10}$

In order to find out how stable vergence control develops in normal, as opposed to dyslexic, children we have examined over 750 primary school children orthoptically and sought correlations between their binocular control and their reading. ${ }^{6.11}$ Only 54 per cent of 5 year old children show stable vergence control in the Dunlop Test but as each year goes by approximately 10 per cent more children acquire it. In support of our hypothesis we found that both within each year group, and on pooling all years together, children who had acquired stable responses in the Dunlop Test were significantly better readers than those who had not. Thus there was a strong tendency for those with unstable vergence control to be backward readers, and those with accurate vergence control to be good readers. ${ }^{11}$

In order to show that the acquisition of fine binocular control leads to improved visual direction sense for small targets, and that this is followed by easier reading, we have been attempting to improve dyslexic children's vergence control. We have used the technique of monocular occlusion. The rationale is as follows: We believe that a dyslexic child's visual confusion is the result of inaccurate binocular control; the two eyes are not always pointing at precisely the same point in space. This gives rise to a variable degree of physiological diplopia which is usually ignored. But the two eyes indicate two different and variable possible positions for a single letter; hence the child's confusion. Therefore occluding one eye should simplify the situation, and perhaps help children to gain stable fine vergence control. In a few cases the results of this approach have been dramatic with leaps in reading age 
of 4 years after only 3 months monocular occlusion. ${ }^{4}$ In our first small controlled trial we demonstrated a significant reading spurt in the children we occluded compared with those we did not. ${ }^{5}$ So with the financial help of the Oxford Regional Health Authority we mounted a double blind controlled trial of monocular occlusion in dyslexic children. ${ }^{12}$

One hundrd and forty-eight dyslexics aged between 8 and 12 years were randomly allocated, by an independent person, either plano or monocularly frosted spectacles to wear only for reading and close work. Neither parents nor children were told which type of spectacles were supposed to be effective. Moreover, when the children were seen again their examiners were not informed what type of spectacles had been worn. But after 6 months 51 per cent of those who were initially unstable and had received monocular occlusion acquired stable vergence control whereas only 24 per cent of those who received plano spectacles did so spontaneously. Most of the children whose vergence control improved also began to read better-increasing their reading age by an average of 11.6 months in the first 6 months of observation and a further 11.3 months in the second 6 months, i.e. they gained on average almost an extra year's reading in the year they were observed. But the reading of the children who had received plano spectacles and did not improve their binocular control, advanced by only 5.6 months in the first 6 months, i.e. their reading actually regressed during the period. The children who were given plano spectacles in the first 6 months were then crossed over to monocular occlusion in the following 6 months. Forty-three per cent of these then acquired good binocular control; moreover, their reading improved by an average of 12.3 months in the next year.

Thirty-two per cent of the dyslexic children already had stable vergence. The design of the trial meant that half of these were also given monocular occlusion. But this had the effect of slightly impeding their reading in comparison with the children with good vergence who were given plano spectacles. Thus as we expected, monocular occlusion only helped those with initially poor vergence control, and it may have made matters worse for those who had already developed good binocular fixation.

Our controlled trial showed that monocular occlusion can help between 26 per cent and 51 per cent of dyslexic children with poor vergence (i.e. approximately 15 per cent to 35 per cent of all dyslexics) to gain good binocular control, and thereby may help many of them to learn to read. However, it has been suggested that we have got cause and effect the wrong way round. Maybe children develop stable vergence control as a result of learning to read, rather than vice versa. We think this most unlikely for 3 reasons.

(1) Acquisition of accurate fine vergence control improves localisation of all small objects, not just letters, so that it is not specific to reading.

(2) Reading improvements follow rather than precede the development of accurate binocular control.

(3) Most of the children who received plano spectacles in the first 6 months of our trial failed to improve their reading and failed to improve their vergence; yet when they were given monocular occlusion in the second six months 45 per cent acquired accurate binocular control and thereafter their reading improved.

It seems that stable vergence control is necessary for accurate visual direction sense and therefore that it is an essential prerequisite for successfully learning to read.

The Dunlop Test is not ideal, however. It requires young children to report their perceptual experiences accurately in a complicated situation. It therefore produces a high proportion of false positives and negatives, particularly in inexperienced hands. Hence there are some reports which fail to show any relationship between Dunlop Test responses and reading retardation. ${ }^{13}$ Accordingly, over the last two years we have been attempting to develop more objective means of assessing vergence control in children. We measure the eye movements of normals and dyslexics during dynamic vergence stress in the synoptophore, using an infrared technique. We have repeated many of the observations described above, using eye movement recordings rather than the subjective responses of the Dunlop Test. We have found an overall 
A

A.McD.

\section{$22^{\frac{k}{0}}$ fusion targets}

\section{Divergence Convergence}

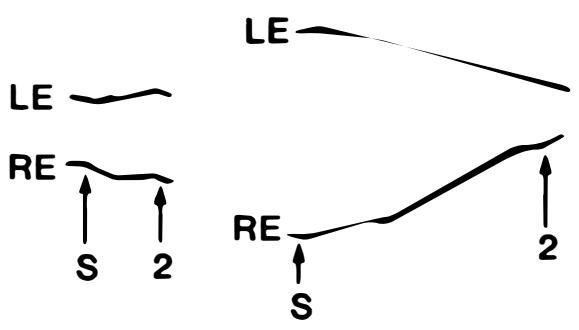

$7^{\circ}$ fusion targets

\section{Convergence}

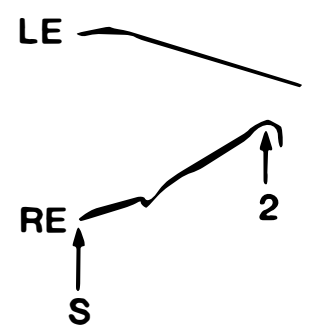

B WP

\section{Divergence Convergence}

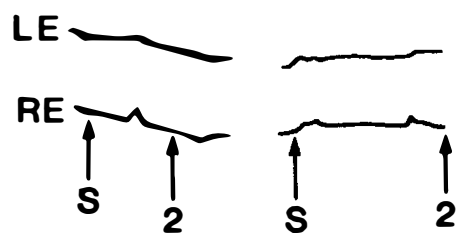

$7^{\circ}$ fusion targets

Convergence

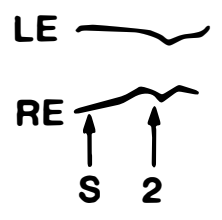



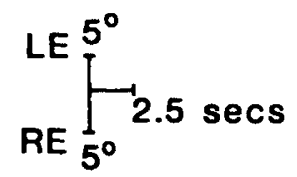

Fig.1a. Normal reader-vergence eye movements tracking macular (2.5 degrees) and large (7 degrees) fusion targets.

Fig. 1b. Dyslexic subject-attempts at vergence. Note inappropriate conjugate eye movements.

Key: LE-left eye. RE-right eye. $s-$ start of stimulus. 2-point at which subject reports diplopia.

concordance of 75 per cent between Dunlop Test and eye movement recordings in 415 subjects. But we believe that the eye movement recordings are more reliable, giving us fewer misleading results.

Using eye movement recordings we confirmed that 67 per cent of dyslexics were unable to make accurate vergence movements in response to the movement of small fusion targets in the synoptophore. ${ }^{14}$ When the synoptophore tubes are converged or diverged, in normal children the two eyes move symmetrically in opposite directions (Fig. 1a). However, 67 per cent of dyslexic children were unable to unyoke their eyes to move in opposite directions under these circumstances. Instead, they moved in parallel, mak- ing inappropriate conjugate movements following just one of the targets; hence the child rapidly experienced diplopia (Fig. 1b). Such poor responses to vergence stress are uncommon in good readers. When we compared the vergence eye movements of dyslexic children with younger good readers who had the same reading age as the dyslexics, 67 per cent of the dyslexics had poor vergence control as shown in Figure 1b; but none of the good readers did, even though they were much younger. Thus we have confirmed our conclusion from the results of the Dunlop Test, directly by vergence eye movement recording, that a high proportion of dyslexics have poor fine vergence control, whereas few good readers have. 


\section{Effect of Occlusion on the Eye Movements}

of a Dyslexic Subject.

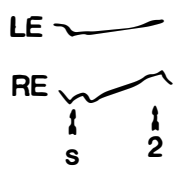

Before Occlusion

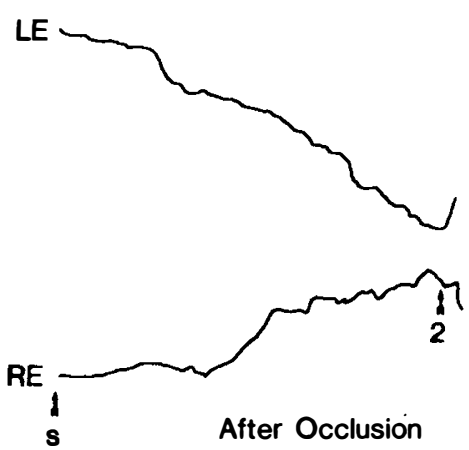

Fig. 2. Effect of occlusion on vergence eye movements in a dyslexic subject. Note inappropriate conjugate movements before occlusion, and much improved fusion range (30 degrees convergence) after 3 months occlusion.

Key: LE-left eye. RE-right eye. s-start of stimulus. 2-point at which subject reports diplopia.

We have also confirmed by recording vergence eye movements during synoptophore tests that monocular occlusion may improve dyslexics' vergence control. Figure 2 a shows the poor convergence eye movements of a 9 year old dyslexic boy. He made the inappropriate conjugate movements described earlier. Hence he was only able to tolerate 3 degrees convergence of the synoptophore tubes before becoming diplopic. His reading age was then 30 months behind his chronological eye. Figure $2 \mathrm{~b}$ shows how his convergence responses had improved after 3 months wearing left monocular occlusion for reading and close work. Though still less smooth than normal, he was now able to make up to 30 degrees appropriate convergent eye movements in response to the vergence stimulus before becoming diplopic. During that time his reading improved by over 2 years, so that he now read at the level expected of his chronological age. The average improvement in reading age in 6 months, which followed acquisition of good vergence responses with the help of monocular occlusion in 23 dyslexic children whose eye movements we have recorded before and after, was 13.6 months. This is similar to the results reported previously. ${ }^{5.12}$

Good binocular control is not only a necessary prerequisite for accurate localisation of small targets. It is probably also essential for fine stereopsis. Using the Randot test we have been measuring the stereoacuity of dyslexic and normal children to compare with their vergence eye movements. As expected, children with poor binocular fixation were found to have significantly poorer stereoacuity than normal children with good vergence control. The average stereoacuity of 33 normal readers was $27 \pm 2.1$ (SE) seconds of arc, whereas that of dyslexics with unstable vergence control was much worse ( $74 \pm 15$ secs). Thus it seems that immature vergence control not only leads to unreliable localisation of small objects such as letters, but also to poor stereoacuity. Happily we have found that when we are successful in improving these children's vergence control, their stereoacuity improves rapidly also.

Our hypothesis is that many dyslexic children have poor control of binocular fixation leading to inaccurate perceptual localisation of small targets, hence difficulty with reading. We have shown that poor vergence control is found in 67 per cent of dyslexic children; but that it is uncommon in good readers; that it leads to reduced stereoacuity; and that when monocular occlusion improves a child's vergence control it also improves stereoacuity and reading. Thus we believe our hypothesis to be well supported by the evidence.

However, impaired ocular motor control is by no means the whole explanation for dyslexia. Thirty-three per cent of dyslexics exhibit normal vergence control. It is these children who make mainly phonological rather than visual errors. ${ }^{12.15}$ Furthermore, 
another 33 per cent of dyslexics are not helped by monocular occlusion to improve their vergence control or to learn to read. These children probably suffer both ocular motor and phonological problems. Clearly if there is a single fundamental cause of dyslexia it can exert its effects in at least two ways-namely by disturbing fine vergence control and/or by disturbing phonemic segmentation. An attractive, though highly speculative, idea is that since fine vergence control is probably a function of the right hemisphere and phonological analysis is the responsibility of the left, the root cause of dyslexia may be a biologically based disturbance of the orderly specialisation of either or both cerebral hemispheres.

\section{References}

${ }^{1}$ Berger M, Yule W, Rutter M: The prevalence of specific reading retardation. Br. J. Psychiatry 1975; 126: 510-9.

${ }^{2}$ Benton AL: Dyslexia-an appraisal of current knowledge. Oxford: Oxford University Press, 1978; 61-112.

${ }^{3}$ Galaburda AM, Kemper TL: Cytoarchitectonic abnormalities in developmental dyslexia. Ann. Neurol. 1979; 6: 94-100.
${ }^{4}$ Fowler MS, Stein JR: New evidence for visual ambilaterality in some dyslexics. Br. Orthoptic J. 1980; 37: 11-15

${ }^{5}$ Stein JR, Fowler MS: Visual dyslexia. Trends Neurosci. $1981 ; 4$ : 77-80.

${ }^{6}$ Stein JR, Fowler MS: Ocular motor dyslexia. Dys lexia Rev. 1982; 5: 25-8.

${ }^{7}$ Stein JF, Fowler MS: Ocular motor problems of learning to read. In: Gale AG, Johnson F, eds. Theoretical and applied aspects of eye movement research. Elsevier: Amsterdam, 1984; 251-9.

${ }^{8}$ Dunlop P: Dyslexia. The orthoptic approach. Austr. Orthopt. J. 1972; 12: 16-20.

${ }^{9}$ Stein JF, Fowler MS: Diagnosis of dyslexia by means of a new indicator of eye dominance. $B r . J$. Ophthalmol. 1982; 66: 332-6.

${ }^{10}$ Bigelow ER, McKenzie BE: Unstable ocular dominance and reading. Perception 1985; 14: 329-35.

${ }^{11}$ Stein JF, Riddell PM, Fowler MS: The Dunlop test and reading in primary school children. Br. J. Ophthalmol. 1986; 70: 317-20.

12 Stein J, Fowler MS: Effect of monocular occlusion on visuomotor perception and reading in dyslexic children. Lancet 1985; pp 69-73.

${ }^{13}$ Newman SP, Wadsworth JF, Archer R, Hockly R: Ocular dominance, reading, and spelling ability in school children. Br. J. Ophthalmol. 1985; 69: 228-32.

${ }^{14}$ Stein JF, Riddell PM, Fowler MS: Disordered vergence control in dyslexic children. $\mathrm{Br} . \mathrm{J}$. Ophthalmol. 1986 (in press).

${ }^{15}$ Bryant P, Bradley L: Children's Reading Problems 1985; Blackwells: Oxford. 